site of its discovery at Nariokotome in Kenya. A further source of proxy data is the working of ancient flint-knapping sites: the products of left-handed knappers are recognizable as such, as is the pattern of wear on tools used by left-handed early people.

This is not as irrelevant to the workshop's hidden agenda as it may seem. Thus, Crow described his analysis of the 11,600 children tested for hand skills and various cognitive abilities at age 11 in the British National Child Development Study; the striking conclusion is that there is a marked (and statistically significant) deficit of performance on four tests among those with equal hand skills, called 'the point of hemispheric indecision'. Ambiguity about handedness seems to be an innate disadvantage (at least at the age of 11). The same group of children is also a commoner source of schizophrenic breakdown later in life than would be expected.

So what is the gene? Its function, says Crow, must be to induce the development of the cerebral asymmetry that usually puts Broca's area on the left; a mutation would then account for its being sometimes on the right. Post-mortem studies, and now magnetic resonance imaging, suggest that people with schizophrenia tend to lack pronounced asymmetry, making the high prevalence of the disease (apparently unreduced by natural selection despite the reduced fertility of people with schizophrenia) part of the price that Homo sapiens has to pay for the faculty of language.

And where is the gene? Crow's suspicion that it is X-linked is supported by the work of D. H. Skuse (Univ. College, London), who told the workshop of his work with patients suffering from Turner's syndrome (single-X females; see Nature 387, 705-708; 1997) which suggests that a maternally imprinted $\mathrm{X}$-chromosome gene is responsible for their measurable cognitive impairment. From this and other studies, Crow concludes that there is a gene responsible for developmental asymmetry near the centromere of the $\mathrm{X}$ chromosome, and also on the Y. One suspects he will not be the only one looking for it in the years ahead. But only zealots believe that such a tangled tale can have such a simple resolution.

John Maddox is Emeritus Editor of Nature.

\title{
Trees to trap photons
}

Shaul Mukamel

$\square$ hotochemical reactions are usually triggered by a high-energy ultraviolet or visible photon that delivers the necessary energy. In some cases such reactions can instead be induced by the absorption of many low-energy infrared photons using intense infrared lasers. But on page 454 of this issue ${ }^{1}$, Jiang and Aida report a remarkable observation that could lead to a new strategy for multiphoton chemistry. They attach a photoisomerizable molecule to the centre of a spherical cavity formed by a highly branched molecule, or dendrimer. Low-power infrared light then triggers the cis- to trans- isomerization (twisting the molecule around the nitrogen-nitrogen double bond).

In chemical reactions, vibrational energy affects rates and branching ratios among the various products ${ }^{2}$. Unimolecular reactions such as isomerization are usually slow compared with vibrational energy flow within a molecule - vibrational energy is typically redistributed in less than a picosecond. Such reactions can therefore be described using statistical theories which assume that vibrational energy is distributed thermally among the various modes.

To induce a reaction, enough energy is needed to break a molecular bond. A single visible or ultraviolet photon is enough, but infrared photons carry a relatively small amount of energy, so in order to undergo the same reaction a molecule needs to absorb a large number of infrared photons.

The dream of multiphoton laser chemistry has been to pump many quanta of light into a single mode (or bond) using high-power lasers, with the hope that the particular bond will break before the energy can dissipate to

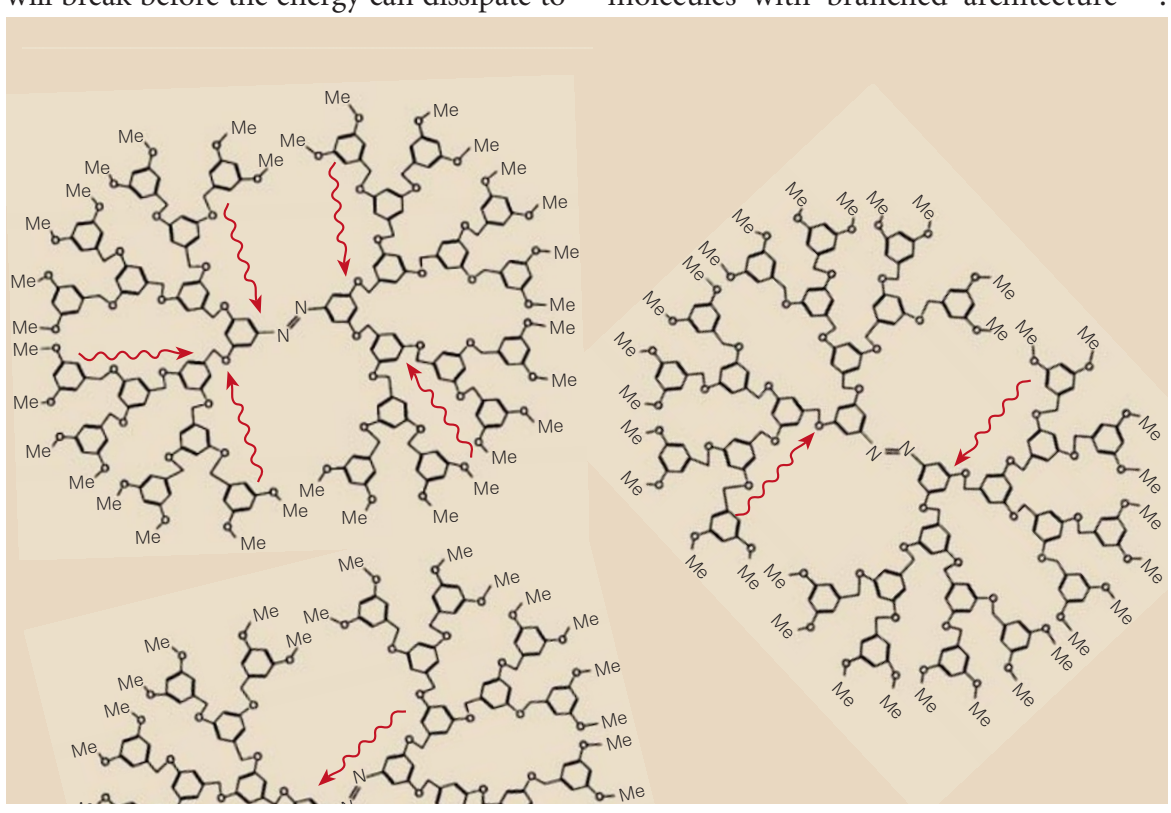

Figure 1 Three dendrimers in solution. Vibrational excitations are induced by an infrared laser, and funnelled towards the centre of each molecule. One contains five excitations - just enough energy in total to isomerize this molecule. Because they are prevented from leaking into the solvent by the close-packed, rigid outer shell, these excitations with eventually combine to cause the isomerization. other degrees of freedom. The prospect of using high-power infrared lasers to do this created great excitement in the mid-1970s ${ }^{3}$. Initial reports seemed very promising. $\mathrm{A} \mathrm{CO}_{2}$ laser, with a power of $\sim 20 \mathrm{MW} \mathrm{cm}^{-2}$, was able to break a S-F bond in $\mathrm{SF}_{6}$ (a 30- to 40-photon process). But in fact this was a thermal reaction: coherent pumping of energy could not compete ${ }^{3}$ with vibrational energy flow. The net effect of infrared laser pumping was incoherent heating, and the laser turned out to be an expensive Bunsen burner.

How could this redistribution be avoided? One group proposed decoupling a large molecule into segments using a heavy atom, in order to limit vibrational energy redistribution ${ }^{4}$. In recent years the focus of laser chemistry has shifted to the use of carefully crafted laser pulses with precise envelopes, timing and even phases to achieve selectivity and control ${ }^{2,5,6}$. But so far, selective, nonthermal multiphoton laser chemistry has only worked in very small molecules with well-separated energy levels.

Another way to increase reaction rates would be if vibrational energy could somehow be channelled into a specific reactive site or bond. Ordinary random fluctuations may take a long time to get the energy to the right place. Some bias, such as an external field or energy gradient, could direct the energy flow $^{7-9}$. Alternatively, coherent migration of vibrational energy among the chromophores of a branched molecule could concentrate enough energy on a shorter timescale.

The new experiment ${ }^{1}$ is based on this principle, but it turns out that the type of molecules used not only funnel the energy usefully, they stop it leaking out. Dendrimers are an interesting class of tree-like macromolecules with branched architecture ${ }^{10,11}$.

(3) 
The dendrimeric backbone contains a large number of aromatic benzene rings, each of which can be excited into a stretching vibration by an infrared photon. With low light intensity, no one ring gets excited by many photons, but the absorption of a single photon by several rings puts enough energy in the dendrimer to make an isomerization possible. Intramolecular funnelling of the energy to the reaction site appears to be the rate-limiting factor. This is reminiscent of thermally activated unimolecular reactions at low pressures, where intermolecular activation determines the rate ${ }^{12}$.

No attempt is made to coherently pump the energy, and the absorption of light is strictly linear. The role of the dendrimeric structure is twofold. First, it serves as a lightharvesting device that collects many excitations close to the reactive site. Second, it maintains these excitations for a long time by slowing the escape of vibrational energy to the surrounding medium. In a dendrimeric geometry the density of atoms increases rapidly with size (or branching generation) and the atoms at the boundary become closely packed, hindering the motions that lead to the loss of energy. The trapped excitations then have plenty of time to explore phase space (that is, to be randomly redistributed between bonds) and eventually combine in the desired region of the molecule where the reaction takes place.

This picture of an unconstrained interior protected by a rigid boundary is supported by nuclear magnetic resonance measurements. (The degree of rigidity may depend on the type of dendrimer and the solvent's quality, as shown by molecular dynamics simulations ${ }^{13}$.) Further evidence for this process is provided by the absence of reactivity when the reactive site is on the outside rather than the inside of the dendrimer. Moreover, the increase in reactivity is observed only in highgeneration dendrimers, where the exterior is closely packed and therefore stiff.

The reported rate scales with the fifth power of light intensity. This can be explained as follows: if each dendrimer has $N$ benzene rings and if a fraction $s$ of the rings has been excited (on average, among many molecules), then the probability that a particular dendrimer will carry $n$ vibrational quanta is the poissonian $P(n)=\mathrm{e}^{(-N s)}(N s)^{n} / n !$. Because $s \ll 1$, only a small fraction of the molecules will acquire the necessary energy; but all these molecules will eventually react, as the energy does not dissipate. The fraction $s$ is proportional to the light intensity and the $s^{n}$ factor therefore implies $n$th power dependence on the light intensity. The azobenzene system needs five photons to induce the reaction, which explains the $n=5$ observed dependence on incident power. (In the multiphoton laser experiments, the nonlinear dependence on incident power reflects the nonlinear absorption of light; here the absorption is strictly linear, and the nonlinearity comes from the need to create many excitations in the same molecule.)

Similar funnelling of energy occurs naturally in photosynthesis, where assemblies of chlorophyll antennae absorb energy and direct it to a reaction centre, where a primary electron transfer takes place. Dendrimeric geometry is very attractive as a possible artificial antenna ${ }^{14}$ - the absorbing units can be closely packed without much support structure, and a simple chemical change of the central group may induce a large conformational rearrangement of the dendrimer ${ }^{15}$. So this new-found double role of dendrimers, as light-harvesting antennas as well as prisons for vibrational energy, may allow new forms of supramolecular photochemistry.

Shaul Mukamel is in the Chemistry Department, University of Rochester, PO Box RC 270216,

Rochester, New York 14627-0216, USA.
1. Jiang, D.-L. \& Aida, T. Nature 388, 454-456 (1997).

2. Rice, S. A. in Proc. 20th Solvay Conf. Chemistry (ed. Gaspard, P.) Adv. Chem. Phys. Vol. 101 (Wiley, New York, in the press).

3. Black, J. G., Yablonovitz, E., Bloembergen, N. \& Mukamel, S. Phys. Rev. Lett. 38, 1131-1134 (1977).

4. Lopez, V., Fairen, V., Lederman, S. M. \& Marcus, R. A. J. Chem. Phys. 84, 5494-5503 (1986).

5. Warren, W. S., Rabitz, H. \& Dahleh, M. Science 259, 1581-1589 (1993)

6. Mukamel, S. Principles of Nonlinear Optical Spectroscopy (Oxford Univ. Press, New York, 1995).

7. Kopelman, R. et al. Phys. Rev. Lett. 78, 1239-1242 (1997).

8. Bar-Haim, A., Klafter, J. \& Kopelman, R. J. Am. Chem. Soc. 119, 6197-6198 (1997).

9. Devadoss, C., Bharathi, P. \& Moore, J. S. J. Am. Chem. Soc. 118, 9635-9644 (1996).

10. Newkome, G. R., Moorefield, C. N. \& Vögtle, F. Dendrimeric Molecules (VCH, Weindheim, 1996).

11. Tomalia, D. A., Naylor, A. M. \& Goddard, W. A. Angew. Chem. Int. Edn Engl. 29, 138-175 (1990).

12. Robinson, P. J. \& Holbrook, K. A. Unimolecular Reactions (Wiley, New York, 1972).

13. Murat, M. \& Grest, G. S. Macromolecules 29, 1278-1285 (1996)

14. Gust, D. Nature 386, 21-22 (1997).

15. Junge, D. M. \& McGrath, D. V. Chem. Commun. 857 (1997)

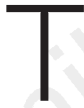
hose who study learning and memory generally agree that many forms of these processes are due to long-lasting, use-dependent changes in the strength of synapses in the brain. Depending on the pattern of synaptic activation, synapses can show either a long-term potentiation (LTP) or a long-term depression (LTD). Both forms of plasticity are spatially restricted that is, they seem to be limited only to those synapses in which the plasticity has been induced. This synapse-specificity has been used in neural-network models that store information, and it suggests that the signals responsible for the changes in synaptic strength are highly localized.

Now, an article by Fitzsimonds et al. ${ }^{1}$ (page 439 of this issue) indicates that a form of LTD may spread over hitherto unimagined distances. Using simultaneous recordings from three interconnected neurons in culture, the authors show that LTD is not limited to the synapses at which it is induced, but that it spreads to other synapses in the microcircuit. They propose that this spread involves signals travelling retrogradely across the synapse and, in addition, signals that travel intracellularly for considerable distances. Moreover, although the changes are widespread, specific rules determine which synapses are affected (Fig. 1, overleaf).

The idea that $\mathrm{LTD}^{2-6}$ and $\mathrm{LTP}^{7-9}$ may not be strictly synapse-specific is not entirely new. So why is the paper by Fitzsimonds et al. ${ }^{1}$ so provocative? First, it challenges the classic view - as originally put forward by Cajal — that the neuron is 'dynamically polarized'; that is, the information received by the dendrites is passed forward through the axon to the synapses on the next cell. The newly discovered backward-propagation of LTD indicates that there is also an intracellular, retrograde, axon-terminal-to-dendritic flow of information (on a much slower timescale). In this way, the input of the neuron is 'told' the state of the output of the neuron. So dendrites not only integrate synaptic potentials, but they also integrate retrograde signals from various axonal outputs of the cell. This has important implications for models of learning that are based on backpropagation algorithms ${ }^{10}$.

Second, the cell-biological mechanisms that must be proposed to account for many of the observations go far beyond those previously considered. Earlier work ${ }^{2-5,7-9}$ indicates that synaptic plasticity may spread laterally via a signal in the extracellular space. But Fitzsimonds et al. suggest that a retrograde signal (at synapse 2 in Fig. 1) generates a secondary intracellular signal in the presynaptic cell. This signal must travel all the way back into the dendrites (to depress synapse 1) and also throughout the presynaptic axonal arbour (to depress synapse 5). So this signal can depress synapses, whether it reaches the synapse from the presynaptic or postsynaptic side. The same, or a different, signal produced by excitatory or inhibitory synapses spreads LTD through the dendrites of the postsynaptic cell to other inputs that converge onto the cell (to depress synapse 4), yet it is incapable of forward propagation down the axon (to synapse 3). All in all, this is a level of complexity that few would have ever considered.

What remains to be done? As is the case with most provocative studies, far more questions are raised than answered. First, how far 